\title{
Das genetische Risiko halbieren
}

\author{
Diabetes und Herz-Kreislauf-Erkrankungen im Zusammenspiel
}

Wer sich gesund ernährt, nicht raucht, körperlich aktiv ist und Übergewicht vermeidet, hat ein um etwa fünfzig Prozent geringeres Risiko für Herz-Kreislauf-Erkrankungen. Das gilt auch für chronisch Erkrankte wie Diabetiker.

Das durch Lebensstilmaßnahmen reduzierte Risiko liegt immer vor, also auch unabhängig davon, ob eine hohe oder niedrige genetische Disposition vorliegt. Dies ist das Ergebnis einer großen Untersuchung mit mehr als 55.000 Teilnehmern. Die Studie unterstreicht die Notwendigkeit nach einer Verhältnisprävention für chronische Erkrankungen, stellt die Deutschen Diabetes Gesellschaft (DDG) fest. Aufgabe der politisch Verantwortlichen müsse sein, die Lebensbedingungen so zu gestalten, dass gesundes Verhalten gefördert werde - etwa durch eine Umstrukturierung der Mehrwertsteuer für Lebensmittel oder einer täglichen Stunde Sport für Kinder in Kindergarten/Kita und Schule.

\section{Häufigere Herz-Kreislauf- Erkrankungen bei Diabetes}

„Diabetespatienten leiden zwei- bis dreimal so häufig an Herz-Kreislauf-Erkrankungen wie andere Menschen, und Herzinfarkt und Schlaganfall sind bei ihnen die häufigste Todesursache“, erklärt DDG Präsident Prof. Dr. Baptist Gallwitz vom Universitätsklinikum Tübingen. Der Grund ist eine beschleunigte Verkalkung der Blutgefäße, für die nicht allein der erhöhte Blutzuckerspiegel verantwortlich ist. „Die meisten Menschen mit Typ-2Diabetes haben auch veränderte BlutfettWerte, Bluthochdruck und massives Übergewicht", sagt Gallwitz, ,eine Konstellation, die zusammen mit dem erhöhten Blutzucker das metabolische Syndrom beschreibt."

Teilnehmer von drei aktuellen Kohorten-Studien aus den USA und Schweden, die ein metabolisches Syndrom hatten, wiesen einen ungesunden Lebensstil auf, der mit einem erhöhten Risiko verbunden war, innerhalb von zehn Jahren einen Herzinfarkt zu erleiden. Bei vielen Teilnehmern der ARIC-Studie (Atherosclerosis Risk in Communities), MDCS-Studie (Malmö Diet and Cancer Study) und

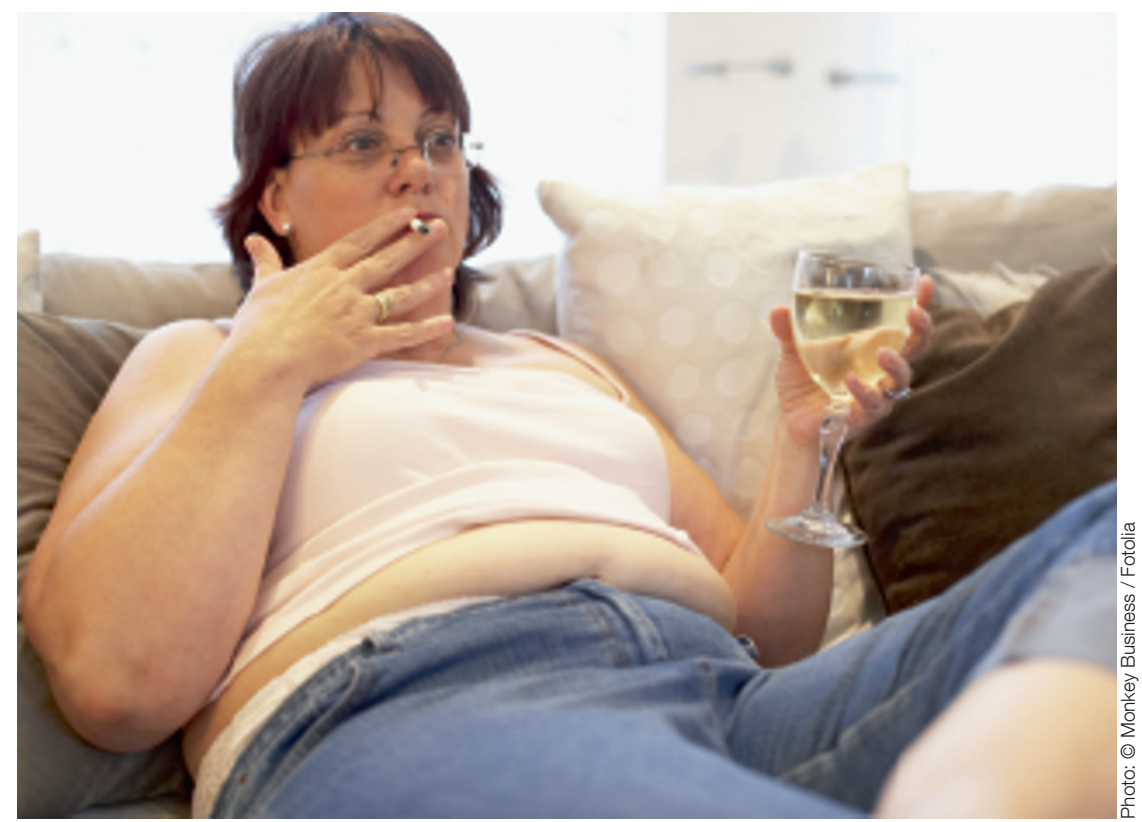

Wer aufhören kann, verbessert in jedem Fall seine Aussichten.

WGHS-Studie (Women's Genome Health Study) kam noch eine genetische Vorbelastung hinzu. Sie wurde mit einem Test ermittelt, der 50 verschiedene genetische Risiken erkennen konnte.

\section{Gesunder Lebensstil wirkt günstig}

Wie erwartet, erkranken Menschen, die nicht rauchen, sich gesund ernähren, körperlich aktiv und nicht fettleibig sind, deutlich seltener an einem Herzinfarkt. „Der günstige Einfluss eines gesunden Lebensstils war in der Studie aber auch bei Menschen nachweisbar, die ein erhöhtes genetisches Risiko hatten", berichtet Gallwitz. Und dieser Einfluss war erheblich, wie die Daten belegen: In der ARIC-Studie erlitten 5,1 statt 10,7 Prozent der Teilnehmer mit einem erhöhten genetischen Risiko einen Herzinfarkt oder andere koronare Erkrankungen. In der WGHS-Studie sank die Rate von 4,6 auf 2,0 Prozent und in der MDCS-Studie von 8,2 auf 5,3 Prozent.. „Die Studie zeigt, dass jeder etwas gegen den Ausbruch einer Krankheit tun kann“, kommentiert Prof. Dr. Dirk MüllerWieland, Vizepräsident der DDG. Staat und Gesellschaft sollten die Menschen aktiv unterstützen. „Wir leben in einer Zeit, in der ungesunde kalorienreiche Nah- rungsmittel oft kostengünstiger sind als gesunde Produkte“, stellt DDG Geschäftsführer Dr. Dietrich Garlichs fest. „Der Staat sollte deshalb die Mehrwertsteuer auf gesunde Lebensmittel senken und für stark zucker- und fetthaltige Produkte erhöhen." Wichtig sei zudem eine tägliche Stunde Sport für Kinder und Jugendliche. „Auch ein Verbot der Tabakaußenwerbung ist längst überfällig“, betont Garlichs: „Wenn wir chronische Krankheiten verhindern wollen, brauchen wir Maßnahmen zur Verhältnisprävention, die einen gesunden Lebensstil fördern.“

Quelle: Presseinformation Deutsche Diabetes Gesellschaft (DDG)

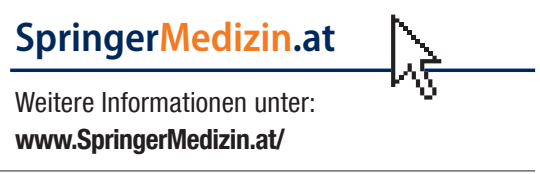

